\title{
Symmetries and special solutions of a parabolic chemotaxis system
}

\author{
Maria Bruzon ${ }^{1}$, Maria Luz Gandarias $^{2}$, Mariano Torrisi $^{3}$, and Rita Tracina ${ }^{4}$ \\ ${ }^{1}$ University of Cadiz, Spain \\ ${ }^{2}$ University of Cadiz \\ ${ }^{3}$ University of Catania \\ ${ }^{4}$ Università di Catania
}

April 28, 2020

\begin{abstract}
In this paper we consider a class of chemotaxis models with two arbitrary constitutive functions $g(u)$ and $f(v)$. After having performed a complete symmetry group classification with respect to them the reduced systems are derived. By considering $\mathrm{g}(\mathrm{u})$ of the logistic form wide classes of exact solutions are found.
\end{abstract}

\section{Hosted file}

FIN_version_20_3_25.pdf available at https://authorea.com/users/306737/articles/437754-symmetriesand-special-solutions-of-a-parabolic-chemotaxis-system 
figures/logistico3/logistico3-eps-converted-to.pdf 
figures/kink2/kink2-eps-converted-to.pdf 
figures/soliton2/soliton2-eps-converted-to.pdf 